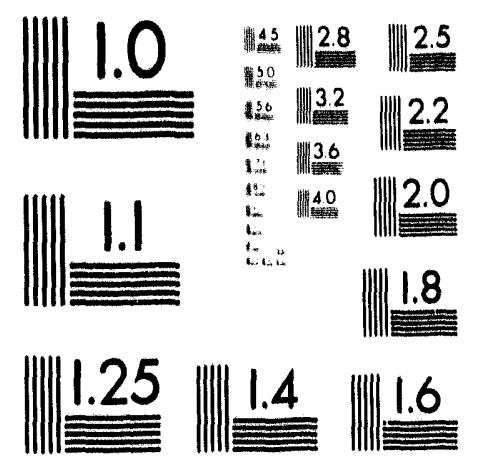



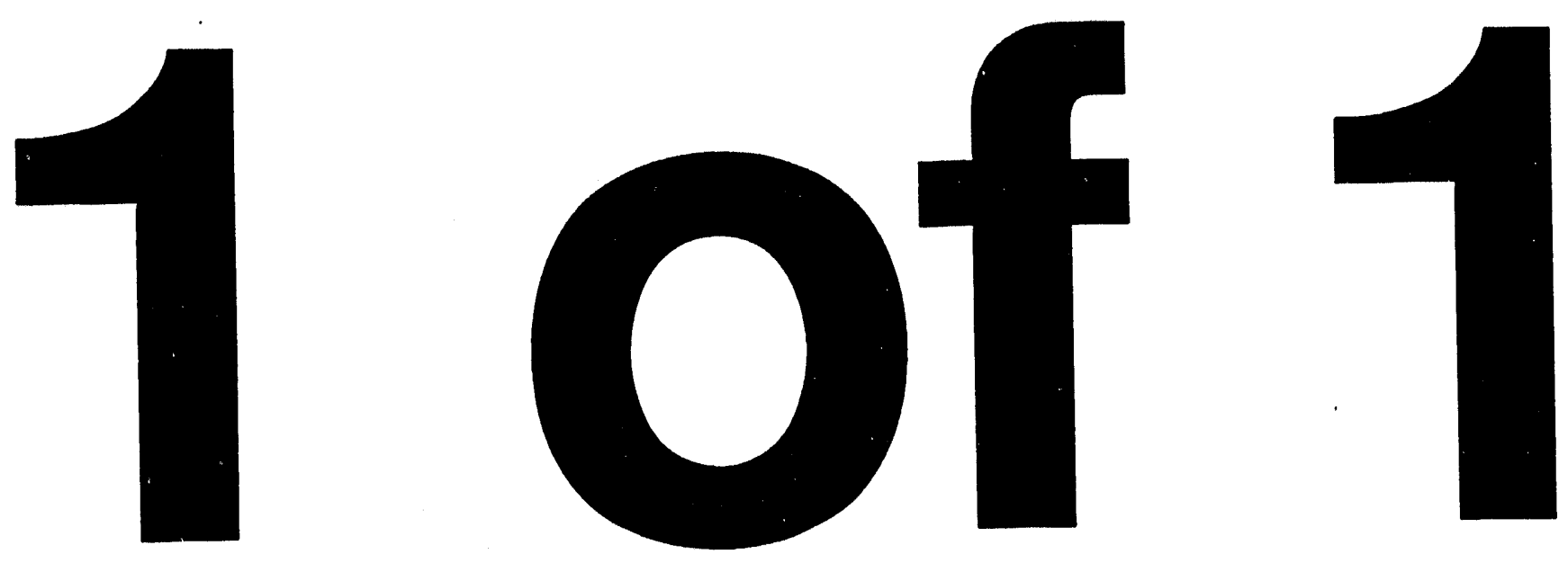
Note: This is a preprint of a manuscript being prepared for publication. Contents of this paper should not be quoted or referred to without permission of the author(s).

Invited paper to be presented at the 1993 International Conference on Advanced Materials, Tokyo, Japan, August 31-Sepember 4, 1993.

\title{
Lattice damage during ion implantation of semiconductors
}

\author{
T. E. Haynes \\ Solid State Division \\ Oak Ridge National Laboratory \\ Oak Ridge, Tennessee 37831
}

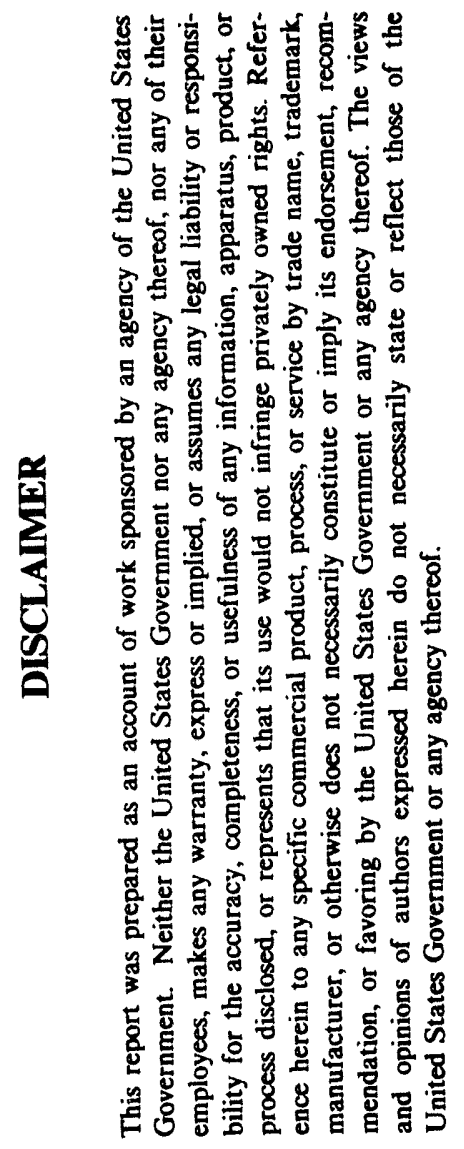

"The submitted manus tript has been authored by a contractor of the US Government under contract No. DEAC05-840R21400. Accordingly, the US Government retains a nonexclusive, royalty-free license to publish or reproduce the published form of this contribution, or allow others to do so, for US Government purposes."

\author{
Prepared by the \\ Oak Ridge National Laboratory \\ Oak Ridge, Tennessee 37831 \\ managed by \\ MARTIN MARIETTA ENERGY SYSTEMS, INC. \\ for the \\ U.S. DEPARTMENT OF ENERGY \\ under contract DE-AC05-84OR21400.
}

August 1993 


\title{
Lattice damage during ion implantation of semiconductors
}

\author{
T. E. Haynes
}

Solid State Division, Oak Ridge National Laboratory, Oak Ridge, Tennessee 37831 USA

The temperature dependence of the lattice damage created during ion implantation of $\mathrm{Si}, \mathrm{Ge}, \mathrm{Si}-\mathrm{Ge}$ alloys, and various III-V compounds is reviewed and interpreted in terms of a transition between two different damage formation mechanisms. The implications of this transition for control of damage, annealing, and electrical activation are discussed, particularly in GaAs.

\section{INTRODUCTION}

Ion implantation continues to be the chief means of introducing controlled amounts of dopants into semiconductors for both device research and development and for circuit manufacturing. The lattice damage created by ion bombardment during the implantation process necessitates high-temperature annealing for eliminating detrimental defects and for electrically activating the implanted dopant atoms. Accordingly, there is a real need to control the damage development during implantation, in order to minimize the thermal budget of post-implantation recovery steps and ultimately to optimize the manufacturing process. Based on experiments which monitor the damage produced as a function of the most important parameters in ion implantation, such as ion species, energy, substrate temperature, and implantation rate, a large number of models for damage evolution have been proposed and evaluated. However, the damage production is inherently a very complex process, as it occurs dynamically in solids which, in terms of the energy density and defect concentration, are extremely far from equilibrium. Consequently, the models which have been developed are not sufficiently accurate to provide good predictions, for instance in process simulators, except in an extremely narrow window of process conditions. Extrapolation is just not possible. In silicon, this deficiency can largely be ignored because the annealing of the predominant defects in silicon is relatively simple and complete recovery is often achievable, so that the final outcome can be somewhat independent of the initial damage state. However, this fortunate circumstance is not likely to hold for much longer. As device dimensions in Si shrink, sensitivity to the few residual defects is likely to increase. Furthermore, in devices based on more complex semiconductor materials, such as alloys, compounds, and heterostructures, where annealing is not quite so simple, the inability to control or predict the initial damage condition over a reasonable processing range is already a technology-limiting barrier.

This paper reviews some recent attempts to define the critical process windows for damage control in a systematic way, and to explore similarities and differences in the damage production in a number of different semiconductors. Those observations allow us to discuss qualititative constraints that an accurate model of implantation damage must obey. Perhaps not too surprisingly, it is clearly necessary to include the dynamic effects of defect interactions during the transient phase of the ion impact, including clustering and recombinationannihilation (dynamic recovery). Experiments have shown that in the most common semiconductor materials the importance of the dynamic interactions grows significantly near room temperature (RT), an unfortunate coincidence. Therefore, understanding these effects vill be critically important if implantation at RT (clearly desirable as the most convenient processing temperature) is to remain viable in the future.

\section{TEMPERATURE DEPENDENCE AND DYNAMIC RECOVERY}

The importance of dynamic recovery in implanted semiconductors is indicated by the temperature dependence of the measured lattice damage in Fig. I for two important families of semiconductor substrates, namely Si-Ge alloys (including the elements $\mathrm{Si}$ and $\mathrm{Ge}$ ) and $\mathrm{GaAs}$. 
The damage in each substrate was measured in the as-implanted condition using ion channeling. The vertical scale in Fig. $I$ is a linear measure of the disorder in the semiconductor crystal at the peak of the damage-depth distribution, and is normalized such that a value of 1 corresponds to complete amorphization. Each substrate in Fig. 1 was implanted with a dose of $1 \times 10^{14} / \mathrm{cm}^{2}$ of Sit at the ion energy which gives a projected range of $100 \mathrm{~nm}$ in that substrate. Details of the original measurements are given in references $1-3$. The key feature illustrated in Fig. 1 is the strong decrease of the lattice damage as the temperature increases. The complete transition from amorphization down to undetectable damage for all of these materials is completely included within a surprisingly narrow temperature range centered roughly around $\mathrm{RT}$ (from $-50^{\circ} \mathrm{C}$ to $+150^{\circ} \mathrm{C}$ ). If the implantation dose is increased, the width of the transition in each substrate becomes narrower, such that the intercept on the temperature-axis remains fixed at a well-defined temperature, which is characteristic of the particular substrate material. In GaAs, for instance, using a dose of $6 \times 10^{14} / \mathrm{cm}^{2}$, the peak damage fraction has been observed to drop from 0.8 to 0.1 over as little as a $10^{\circ} \mathrm{C}$ range between 20 and $30^{\circ} \mathrm{C}$.[1] Such behavior clearly implies that this $\mathrm{T}$-intercept defines a critical temperature for abruptly disabling the primary damage production process in these materials.



Figure 1. Temperature dependence of the damage measured by ion channeling in $\mathrm{Si}, \mathrm{GaAs}$, $\mathrm{Si} 0.65 \mathrm{Ge} 0.35$, and $\mathrm{Ge}$, implanted with $1 \times 10^{14} / \mathrm{cm}^{2}$ of $\mathrm{Si}^{+}$ions (compiled from refs. 1-3).

\subsection{Model for the Temperature Dependence}

An extremely simple model was developed by Morehead and Crowder (MC) more than 20 years ago to describe the increase with implantation temperature of the minimum ion dose for amorphizing Si.[4] According to the MC model, the size of the damaged volume remaining along the track of each impinging ion (on average) is determined by the initial radius, $R_{0}$, of the volume within which defects were originally created by displacement collisions, but reduced by a radius decrement, $\delta R$, equal to the diffusion length of the primary defects migrating out of this volume during relaxation. Assuming thermally activated diffusion, this model leads naturally to the concept of a critical temperature, $T_{0}$, at which the diffusion length becomes equal to the initial radius of the core, so that the residual damage becomes zero. This model predicts that $T_{0}$ will be independent of dose and that the transition will sharpen with increasing dose, as observed. The solid curves in Fig. 1 represent the best-fits of the MC model to data for a range of ion doses.[1-3] Values for the effective diffusivities of the primary defects were obtained from the fitting procedure.

In spite of its extreme simplicity, the MC model nevertheless can make some accurate and useful predictions, for instance, with respect to the dependence of the damage on ion species. As noted, the fitting parameters are interpreted as defining the mobility of the primary defects in the

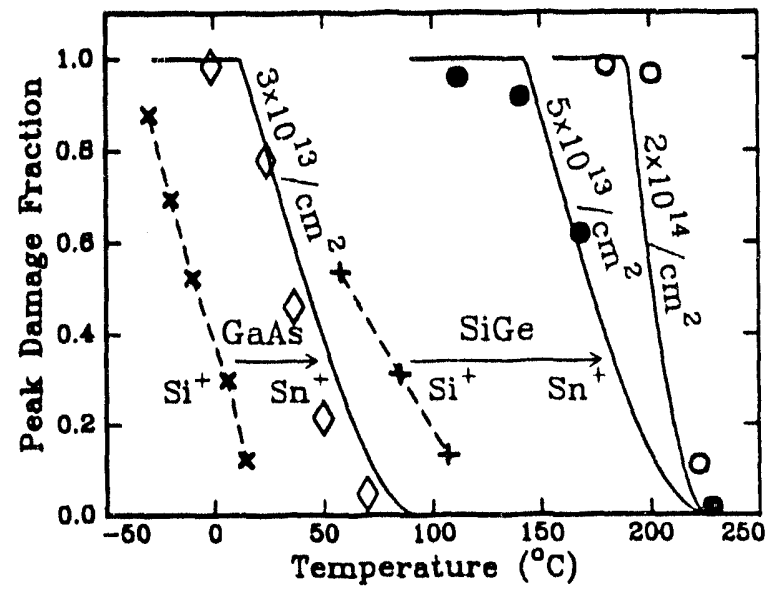

Figure 2. Shift of the transition to higher temperatures for heavier ions is illustrated by a comparison of the damage created by $\mathrm{Si}^{+}$and $\mathrm{Sn}^{+}$ ions implanted into $\mathrm{GaAs}$ and $\mathrm{Si}_{0.65} \mathrm{Ge}_{0.35}$. Predictions for $\mathrm{Sn}^{+}$, based on the $\mathrm{MC}$ model, are indicaled by the solid curves. 
substrate, and therefore do not depend on the ion species. Therefore, having obtained a fit for one ion, the model can be used to predict the temperature dependence for other ion species in the same substrate. Figure 2 illustrates the reliability of such predictions for a couple of cases. An increase in $T_{0}$ is found for ineavier ions since they produced displacements over a larger $R_{0}$, due to the larger rate of energy deposition along the ion's path. The size of this effect can be estimated from the threshold dose for amorphization measured in the limit of low temperatures (no defect out-diffusion) or, alternatively, from the dependence of stopping power on atomic number, Z.[4] In Fig. 2, the transition temperature for $\mathrm{Sn}^{+}(Z=50)$ is compared to that for $\mathrm{Si}^{+}(Z=14)$ ions incident on both $\mathrm{GaAs}$ and $\mathrm{Si}_{0.65} \mathrm{Ge}_{0.35}$. The predicted increases in the transition temperature are approximately $50^{\circ} \mathrm{C}$ in $\mathrm{GaAs}$ and $100^{\circ} \mathrm{C}$ in $\mathrm{Si}_{0,65} \mathrm{Ge}_{0}$ 35. As shown in Fig. 2, the agreement with experiment is quite satisfactory in both cases. Generally, the agreement becomes poorer for lighter ions and low-Z substrates.

\subsection{Dependence on Dose and Dose Rate}

The agreement shown in Fig. 2 supports the argument that the simple $\mathrm{MC}$ model contains a reasonable portion of reality. However, as implied in the introduction, there are a number of observations that cannot be accommodated by such simple models. For instance, this model is strictly linear - it assumes that each ion, on average, produces a fixed amount of additional damage, independent of any pre-existing damage from other ion impacts. Except at low temperatures, or for very heavy ions, damage is usually found not to grow linearly with dose $[5,6]$ i.e., beyond some point, additional ions begin to produce more and more damage, a sign that each ion interacts with defects created by prior ions. Another indication of such interaction is the dependence of the damage on dose rate (implantation beam current), where the amount of residual damage increases when higher beam currents are used. [7] This behavior implies not only that different ions somehow interact, but also that some of the pre-existing defects with which the ions interact are themselves unstable, i.e., given enough time between ion impacts, these defects would effectively anneal during the implantation, but are inhibited from doing so by interaction with defects produced by subsequent ions. Thus, this dose-rate dependence is naturally associated with the dynamic recovery described in Fig. 1. [1,2]

\subsection{Interpretation of Two Regimes}

The dose-rate dependent component of damage is clearly different in nature from the "linear" damage component that dominates at low temperatures. Furthermore, the dose-rate dependent damage becomes dominant at temperatures above $T_{0}$ since the "linear" component vanishes. Therefore, $\mathrm{T}_{0}$ marks the boundary between two different damage growth regimes, i.e., between two different dominant growth mechanisms. At lower temperatures, the damage is dominated by an MC-type, directimpact mechanism and damage growth is quite linear. At higher temperatures, the damage is dominated by reactions between mobile, metastable primary defects produced in different cascades. One manifestation of this is that damage saturation observed in the near-surface region of $\mathrm{MeV}$-implanted $\mathrm{Si}$ and $\mathrm{Si}-\mathrm{Ge}$ alloys occurs only on the high-temperature side of this transition.[8] A strict interpretation of the $\mathrm{MC}$ model would imply that the temperature where this change takes place is controlled by the pointdefect mobility along the ion track. At temperatures near $T_{0}$, both mechanisms are important and the damage growth mode is mixed. Unfortunately, since $T_{0}$ is fairly close to RT for many semiconductors, the damage growth exhibits maximum complexity in most cases for RT implantation. For example, Raman scattering measurements have shown that two different microstructural components contribute to the damage produced in GaAs during implantation at RT.[9]

\section{IMPLICATIONS FOR SiGe ALLOYS}

Note that the transition temperature is higher in Ge than in $\mathrm{Si}$, as shown in Fig. 1, and it has been shown to increase monotonically with $\mathrm{Ge}$ content in the intermediate alloys. $[2,3]$ As a consequence, dynamic recovery at $\mathrm{RT}$ is much more important in $\mathrm{Si}\left(\mathrm{RT}-\mathrm{T}_{0}\right)$ than in $\mathrm{Si}_{1-\mathrm{x}} \mathrm{Ge}_{\mathrm{x}}$ (where $\mathrm{RT}<\mathrm{T}_{0}$ ), and so the damage efficiency (ratio of rinal defect density io displacement denisity) is much greater in the $\mathrm{Si}_{1-\mathrm{x}} \mathrm{Ge}_{\mathrm{x}}$ alloys than in Si. One manifestation of this occurs in implantation of Si/SiGe multilayer heterostructures, where the alloy layers are amorphized preferentially relative to Si layers.[10] 
Although the transition temperature increases monotonically with Ge content, it does not do so uniformly as a function of composition. Most of the temperature shift occurs for Ge fractions less than $40 \%$ [3] From a detailed fitting of the temperature dependences as a function of composition at various $\mathrm{Si}+$ implant doses, it was inferred that the defect mobility effectively reaches a minimum at about $40 \%$ Ge.[3] Consequently, and counterintuitively, the damage efficiency is maximum at this intermediate composition and is even greater than in elemental $\mathrm{Ge}$, although the cascade energy density is larger in Ge.

\section{IMPLICATIONS FOR GaAs}

Among the semiconductors included in Fig. 1, the transition temperature is closest to $\mathrm{RT}$ for GaAs. Therefore, we might expect maximum complexity of damage growth and recovery in GaAs when implanted near RT, due to the mixture of two damage growth modes and the associated combination of damage microstructures.

\subsection{Efrect on Electrical Activation}

In spite of the use of high-temperature, postimplant annealing to recover the lattice damage and activate dopants in GaAs, the electrical activation remains sensitive to subtle differences

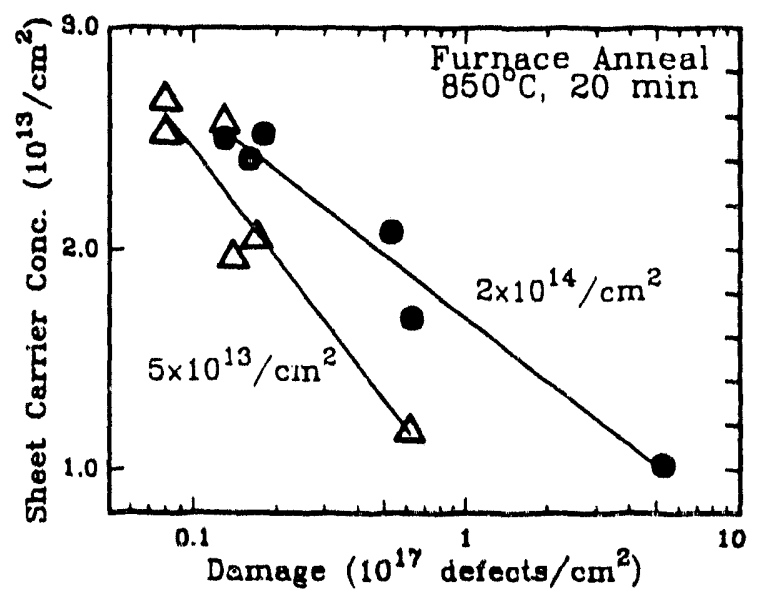

Figure 3. Sheet carrier concentration in GaAs after post-implant annealing is shown as a function of the implant damage before annealing for two different doses of $\mathrm{Si}^{+}$. For each dose, damage was varied by changing the implant temperature and dose rate. in the initial damage condition of the asiniplanted semiconductor.[11] This is illustrated in Fig. 3, where the sheet carrier concentration following post-annealing of Si-implanted $\mathrm{GaAs}$ is shown as a function of the initial defect density measured by ion channeling (integrated over the depth of the profile). In these experiments, the initial damage was varied for a fixed implant dose by changing the implant temperature $\left(+20^{\circ}\right.$ to $\left.90^{\circ} \mathrm{C}\right)$ and the dose rate $\left(0.05\right.$ to $1.0 \mu \mathrm{A} / \mathrm{cm}^{2}$ at RT). These data show that increasing the initial damage has a strong negative imphact on the sheet carrier activation irregardless of the method used to increase the damage. Consequently, the sheet carrier concentration can be improved either by (1) increasing the implantation temperature, or (2) reducing the implantation dose rate. These two approaches are illustrated in Fig. 4. It should be noted that in order to see significant improvement the implant temperature need be raised only by a relatively small amount (up to $90^{\circ} \mathrm{C}$ for normal implantation currents), but the dose rate must be lowered by a significant factor (20x in Fig. 4). Therefore, lowering the dose rate is not a viable approach for improving sheet carrier activation, as it requires a severe reduction in throughput.

\subsection{Pulsed-Beam Experiments}

Since the dose-rate dependence arises from interactions between metastable, mobile defects, the implantation beam current can be applied as a tool for investigating the characteristics of these

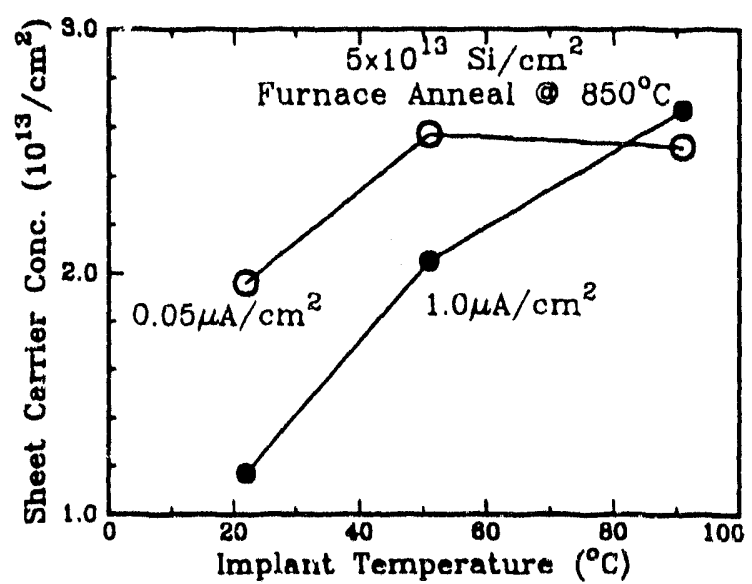

Figure 4. Sheet carrier concentration in GaAs following annealing of $5 \times 10^{15} \mathrm{Si} / \mathrm{cm}^{2}$ implants performed at three temperatures using dose rates of $0.05 \mu \mathrm{A} / \mathrm{cm}^{2}$ (open circles) and $1.0 \mu \mathrm{A} / \mathrm{cm}^{2}$ (filled circles). 
defects. For instance, the dose-rate dependence has been exploited to isolate the two different microstructural components of the damage in GaAs implanted with Si at RT. [9] More recently, a pulsed, focused ion beam (FIB) has been used to probe the time scale of the annihilation of the metastable defects.[12] In these experiments, an FIB approximately $0.2 \mu \mathrm{m}$ in diameter was stepped digitally across a sample area $0.8 \mathrm{~mm}$ in diameter. A number of repetitions were required to reach the necessary implant dose. The dwelltime for each step, and the time between successive implants at the same spot (repetition period) were independently controlled. Representative results are shown in Fig. 5. In this case, the dwell time was fixed at $3.2 \mu \mathrm{sec}$, corresponding to a dose of approximately $1.2 \times 10^{12} \mathrm{ions} / \mathrm{cm}^{2}$ per step. Thus, increasing the repetition period corresponds to reducing the average dose rate. Consequently, the asimplanted damage decreases with increasing repetition period, and the sheet resistance also decreases, matching the trends found in the broad-beam implant case (Figs. 3 and 4). The surprise here is that the dependence of the damage on the repetition period continues over all accessible time scales. This necessarily implies that some of the defects annihilate on time scales much shorter than $\sim 10^{-3} \mathrm{sec}$, while others have lifetimes much longer than $1 \mathrm{sec}$. (Over any time scale on which there were no appreciable defect annihilation, the background defect population

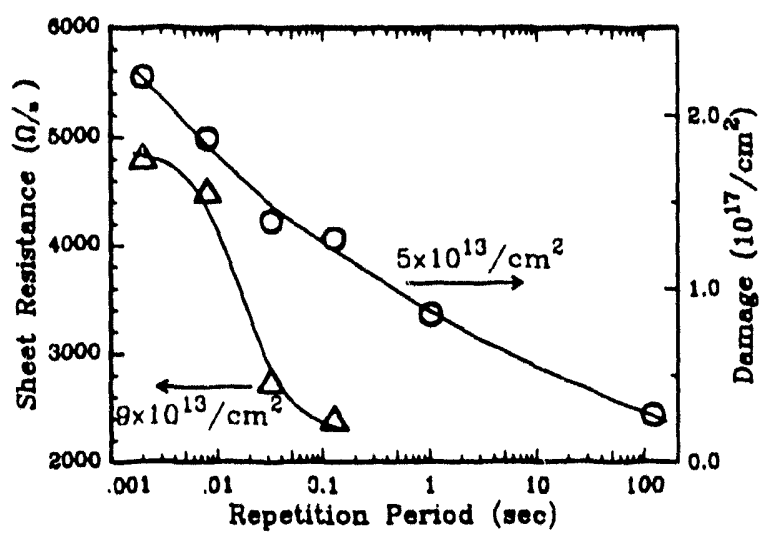

Figure 5. As-implanted damage (circles, dose $\left.=5 \times 10^{13} / \mathrm{cm}^{2}\right)$ and post-annealed sheet resistance (triangles, dose $=9 \times 10^{13} / \mathrm{cm}^{2}$ ) in GaAs following FIB-implantation with $\mathrm{Si}$, as a function of the time interval between passes of the FIB over the sample (adapted from Ref. 12). would be constant and the dependence of damage on repetition period would disappear.) Such behavior clearly adds significantly to the complexity of any physical model which would claim to describe the growth of lattice damage in RT-implanted GaAs. For instance, one anticipated consequence for broad-beam implantation, where the beam is electrostatically or mechanically rastered across the wafer surface, is that the damage and electrical activation should depend on the frequency of the rastering system, which is typically in the range of 10 to $1000 \mathrm{~Hz}$ (corresponding to repetition periods of $10^{-3}$ to $10^{-1} \mathrm{sec}$ in Fig. 5). Such effects have not yet been reported, but ought to be worth checking. (It is possible that the frequency dependence may be mitigated somewhat in broad-beam implants if the raster is intentionally set to be nonrepetitive.)

\section{OTHER SEMICONDUCTORS}

Dynamic recovery is also observed in other semiconductors and, remarkably, it appears in a very similar temperature range for many of them. Figure 6 shows the temperature dependence of lattice damage in Si-implanted GaP and InP[11], and in N-implanted SiC[13] compared to that of Si-implanted GaAs. In the phosphides, the transition occurs at a higher temperature $\left(\mathrm{T}_{0} \sim 100^{\circ} \mathrm{C}\right)$ than in GaAs. Thus, direct-impact damage is more dominant at $\mathrm{RT}$ in the

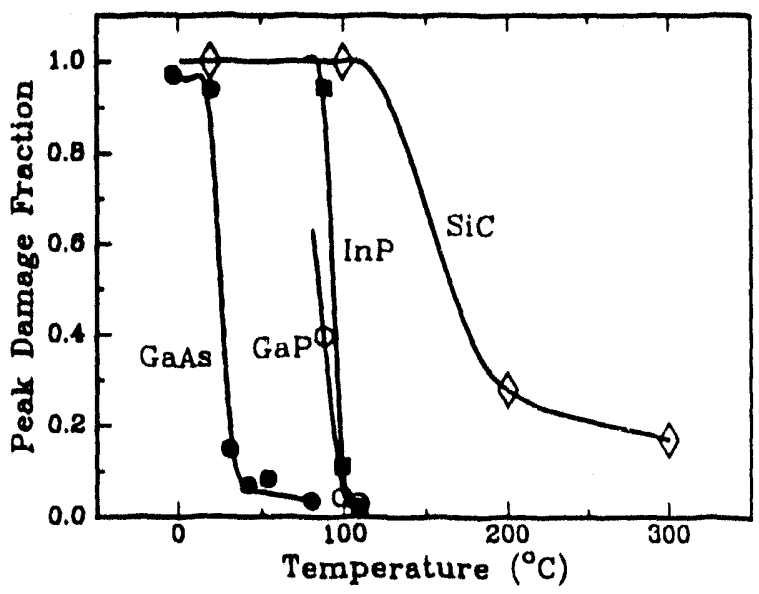

Figure 6. Transition temperatures for GaP and InP implanted with $\mathrm{Si}^{+}$and for $\beta$-SiC implanted with $\mathrm{N}^{+}$are compared to that of $\mathrm{Si}^{+}$. implanted GaAs. 
phosphides than in GaAs, so, by analogy to GaAs, implantation temperatures in excess of $150^{\circ} \mathrm{C}$ ought to yield improved electrical activation as compared to $\mathrm{RT}$, or even $100^{\circ} \mathrm{C}$. Indeed, this appears to be the case.[14] In B-SiC, the data set is somewhat more limited, but points to a change in the damage growth mode below $200^{\circ} \mathrm{C}$. This is a somewhat lower temperature than may be expected based on the more refractory nature of $\mathrm{SiC}$ relative to the other semiconductors. It is not yet clear that this is the same kind of transition as seen in the other semiconductors, because, as shown in Fig. 6, there is a residual damage component at temperatures above $200^{\circ} \mathrm{C}$, even for low dose-rate implants. Recently, investigations of the implantation of oxygen into tin $(5 \%)$-doped indium oxide (ITO) thin films have shown that there is also strong dynamic recovery at $\mathrm{RT}$ in these important semiconductor thin films.[15]

\section{SUMMARY}

In this paper, we have reviewed some recent measurements of lattice damage in a number of ion-implanted semiconductors, including $\mathrm{Si}-\mathrm{Ge}$ alloys, GaAs, GaP, InP, SiC, and ITO, emphasizing the temperature dependence of the damage near RT and the important role of interactions between mobile defects when implants are performed at RT in each of these materials. A critical temperature can be identified where the dominant damage mechanism changes from direct-impact damage formation within individual collision cascades, to agglomeration of mobile primary defects created in separate cascades. In the most important materials (Si and $\mathrm{GaAs}$ ), the transition occurs very near RT (below $50^{\circ} \mathrm{C}$ ) for medium-mass ions, so that damage is produced by a mixture of both modes during implantation at RT. The physical models used in process simulators must include the interactions between primary defects if they are to be reliable in relevant process windows.

\section{ACKNOWLEDGEMENTS}

This work was sponsored by the Division of Materials Sciences, U.S. Department of Energy under contract DE-AC05-840R21400 with Martin Marietta Energy Systems, Inc. Collaborations with R. Morton and S. S. Lau at the University of California-San Diego, and with C. R. Musil and J. Melngailis at the Massachusetts Institute of Technology contributed greatly to this work. Also, discussions with $O$. W. Holland, U. V. Desnica, and Yuzo Shigesato are gratefully acknowledged.

\section{REFERENCES}

1. T. E. Haynes and O. W. Holland, Appl. Phys. Lett. 59, 452 (1991).

2. T. E. Haynes and O. W. Holland, Appl. Phys. Lett. 61, 61 (1992).

3. T. E. Haynes and O. W. Holland, Nucl. Instrum. Methods B 80/81, 901 (1993).

4. F. F. Morehead, Jr. and B. L. Crowder, Radiat. Efr. 6, 27 (1970).

5. O. W. Holland, S. J. Pennycook, and Gerald L. Abert, Appl. Phys. Lett. 55, 2503, (1989).

6. T. E. Haynes and O. W. Holland, Nucl. Instrum. Methods B 59/60, 1028 (1991).

7. T. E. Haynes and O. W. Holland, Appl. Phys. Lett. 58, 62 (1991).

8. O. W. Holland and T. E. Haynes, Appl. Phys. Lett. 61,3148 (1993).

9. U. V. Desnica, J. Wagner, T. E. Haynes, and O. W. Holland, J. Appl. Phys. 71, 2591 (1992).

10. M. Vos, C. Wu, I. V. Mitchell, T. E. Jackman, J-M. Baribeau, and J. McCaffrey, Appl. Phys. Lett. 58, 951 (1991).

11. T. E. Haynes, R. Morton, and S. S. Lau, Mat. Res. Soc. Symp. Proc., volume 300 (in press).

12. C. R. Musil, PhD thesis, Massachusetts Institute of Technology, 1993.

13.S. P. Withrow (unpublished).

14.J. P. Donnelly, Nucl. Instrum. Methods $182 / 183,553$ (1981).

15. Y. Shigesato, D. C.Paine, and T. E. Haynes, in these proceedings. 

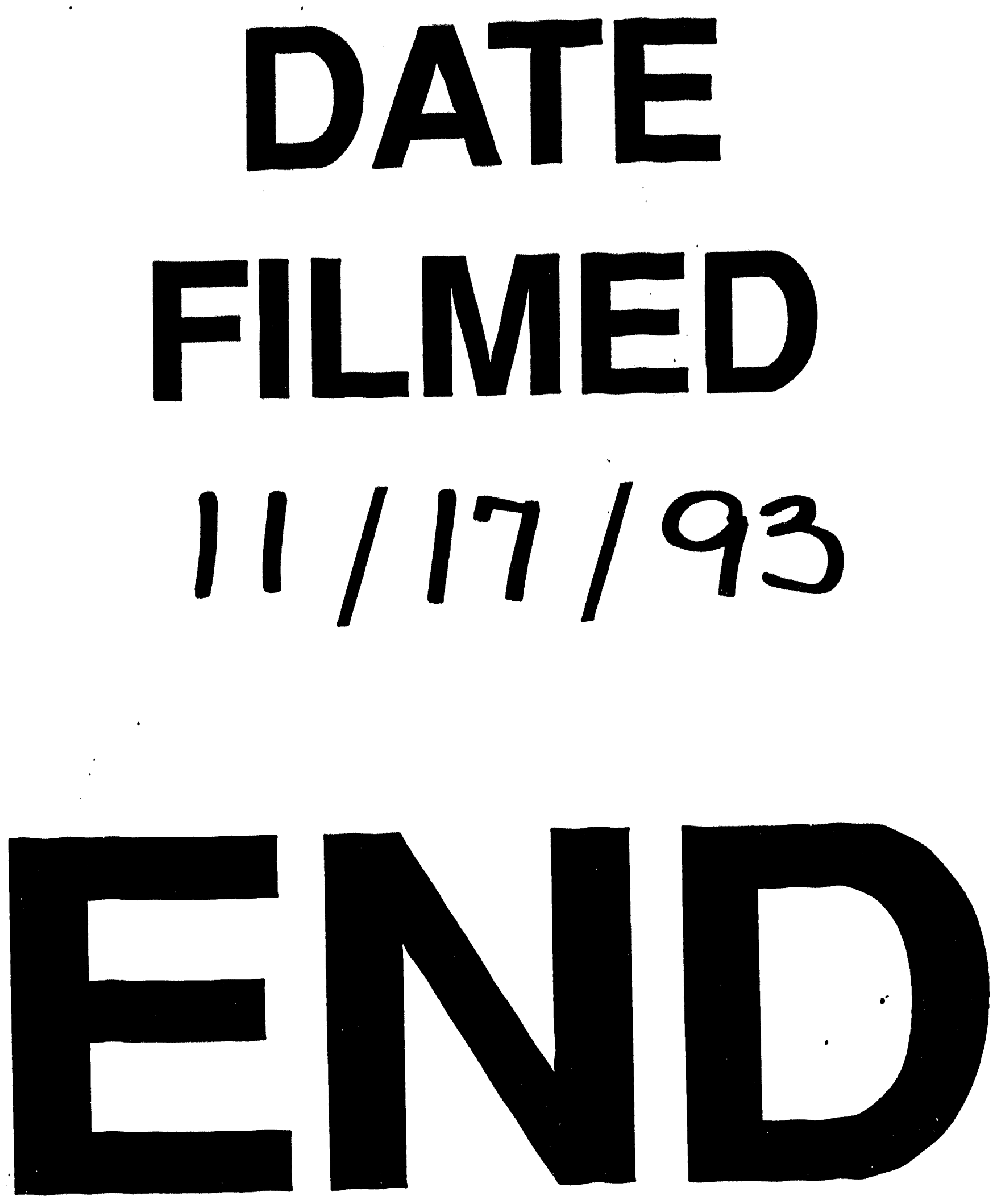




\section{.}

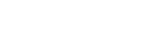

(n)

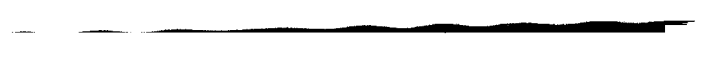

